\title{
The Management Challenges in an Unusual Case of Primary Osteosarcoma of the Rib in an Adult Patient
}

\author{
Arvind Krishnamurthy ${ }^{1}$. Arunandhichelvan Arulmolichelvan ${ }^{1}$
}

Received: 22 July 2015 / Accepted: 10 January 2017 / Published online: 18 January 2017

(C) Association of Surgeons of India 2017

\begin{abstract}
Primary osteosarcomas are derived from primitive bone-forming mesenchymal stem cells and most often originate in the metaphysis of the long bones of children and adolescents, but it occurs very rare in adults. Approximately $10 \%$ of osteosarcomas are located in the flat bones, pelvis being the main site. Involvement of flat bones like rib may be seen as a metastatic process or secondary to chemotherapy, but a primary osteosarcoma rib is extremely rare. Osteosarcoma originating from such rare sites poses numerous diagnostic and therapeutic challenges, and we report one such case in a 24-yearold male patient. Our case highlights the fact that osteosarcoma of the rib, although rare, should be considered in the differential diagnosis of primary malignant neoplasms of the rib even in the adult population. Further, early prompt diagnosis and aggressive multimodality management are cornerstones of management and will help improve the survival outcomes.
\end{abstract}

Keywords Adult osteosarcoma $\cdot$ Rib tumours $\cdot$ Chest wall tumours $\cdot$ Primary osteosarcoma

\section{Introduction}

Osteosarcomas are the most common malignant neoplasms of the bone, the most common site being the metaphysis of the long bones of children and adolescents. Around 10\% of osteosarcomas are believed to arise from the flat bones, the most common site being the pelvis [1]. Involvement of flat bones

Arvind Krishnamurthy

drarvindkrishnamurthy@yahoo.co.in

1 Surgical Oncology, Cancer Institute (WIA), 38, Sardar Patel Rd, Adyar, Chennai 600036, India like rib may be seen as a metastatic process or secondary to chemotherapy, but a primary osteosarcoma rib is extremely rare $[2,3]$. We report a case of primary osteosarcoma arising from the ribs of a 24-year-old male patient highlighting the therapeutic challenges along with a brief review of the literature.

\section{Case Report}

A 24-year-old male patient without any comorbid illnesses was referred to our centre for further evaluation of a recurrent right chest wall swelling of an 8-month duration. He had undergone an attempted excision of the swelling at an outside centre 4 months prior. Clinical examination revealed a painless bony hard fixed mass of $20 \times 15 \mathrm{~cm}$ in the right lateral chest wall with an overlying $10 \mathrm{~cm}$ surgical scar of the prior surgery which had healed by secondary intention. (Fig. 1a) There was no regional adenopathy, and general examination and further systemic examination were normal. A computerized tomography scan of the chest showed the swelling to arise from the fifth to the seventh ribs and with extensive soft tissue involvement including the underlying parietal pleura, the overlying intercostal muscles and the adjoining muscle fibres of serratus anterior, pectoralis major, and the external oblique. Moderate pleural effusion was also noted; the remaining lung parenchyma was however normal. The cytology of the pleural fluid revealed an exudative effusion which was negative for malignant cells. A bone scan revealed an isolated uptake in the fifth, sixth and the seventh ribs (Fig. 1b, c). A review of the histopathology of the initial surgery and the core needle biopsy from the swelling done at our centre confirmed the diagnosis of a high-grade osteosarcoma (Fig. 1d).

The patient after a multidisciplinary board discussion was planned on initial systemic therapy. He received 3 cycles of 
Fig. 1 a A clinical photograph at presentation. b A computerized tomography scan of the chest showing the swelling to arise from the fifth to the seventh ribs and with extensive soft tissue involvement. Also noted is moderate pleural effusion. $\mathbf{c}$ Bone scan revealing an isolated uptake in the fifth, sixth and the seventh ribs. d H\&Ex10: histopathology review of the upfront attempted excision biopsy suggestive of a high-grade osteosarcoma

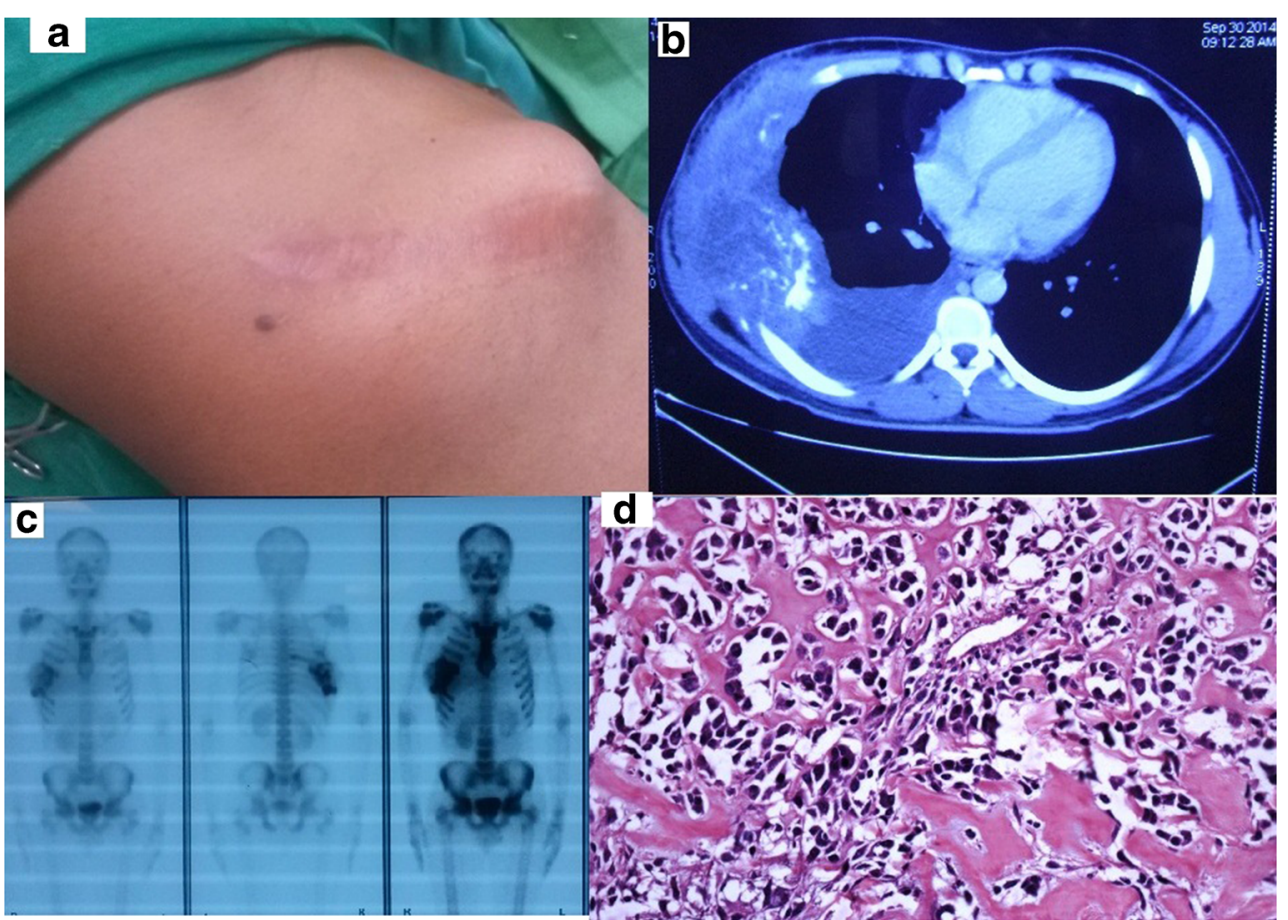

neoadjuvant IAP (ifosfamide, Adriamycin, and cisplatin) chemotherapy. The patient had a static response to the neoadjuvant chemotherapy; a subsequent CT scan of the chest done however showed disappearance of the effusion. The patient was subsequently taken up for a definitive surgery which entailed an en bloc excision of the fifth to the seventh ribs along with the overlying soft tissues and skin. The resultant defect was reconstructed using a Prolene mesh. A latissimus dorsi flap was placed to cover the mesh and the skin was closed primarily.(Fig. 2a-d) The patient had uneventful postoperative recovery; the final histopathology confirmed the diagnosis of a high-grade osteosarcoma with $30 \%$ necrosis extending to surrounding soft tissues with all bony, soft tissue, and skin margins being free of tumour. The patient completed the remaining 3 cycles of adjuvant IAP chemotherapy and is on regular follow-up and is disease free for the past 13 months post-surgery.

\section{Discussion}

Primary osteosarcomas are derived from primitive boneforming mesenchymal stem cells and most often originate in the metaphysis of the long bones. Approximately $10 \%$ of osteosarcomas localise in the flat bones, pelvis being the main site. Primary osteosarcomas of the ribs are infrequent and have been reported mainly in the paediatric population [1-4].

There are no characteristic symptoms or signs of osteosarcoma of the ribs; in fact, primary osteosarcomas originating from flat bones often pose a major diagnostic challenge to the treating clinicians $[5,6]$. The typical 'sunburst' radiological pattern observed in the osteosarcomas of the long bones is generally not observed in osteosarcomas of the ribs and other flat bones. Owing to the exclusive site and varying radiological features, these tumours can be confused with other bone sarcomas including chondrosarcomas and fibrosarcomas. A secondary metastasis from a primary bony osteosarcoma is also an important differential as the two entities have different biological behaviours and require different treatments and ultimately different prognosis. Osteosarcomas typically show increased uptake on technetium-99 $\mathrm{m}$ bone scans. Bone scans are useful in excluding multifocal disease and occasionally skip lesions. Skip lesions are however most reliably identified by a MRI scan [5].

The classic histological feature of a ramifying osteoid matrix laid down by the neoplastic cells with osteoblastic differentiation clinches the diagnosis of osteosarcoma. The diagnosis of a primary osteosarcoma in a small biopsy can at times be challenging. Diagnostic difficulties can additionally be encountered when the osteoid production is scant wherein an extensive sampling of the tumour is warranted. Multiple soft tissue cores easily can be obtained in tumours with extensive soft tissue involvement as was initially performed in our patient. The histological features of certain low-grade osteosarcomas can mimic benign tumours like fibrous dysplasia and desmoplastic fibroma. The histologic pattern differentiating low-grade osteosarcomas from fibrous dysplasia is its permeative growth pattern encasing pre-existing trabecular bone. Extension to the adjacent soft tissues is a feature not associated with fibrous dysplasia. The presence of new bone formation effectively excludes a diagnosis of desmoplastic fibroma. 
Fig. 2 a Operative bed following en bloc excision of the tumour bearing fifth to the seventh ribs along with the overlying soft tissues and skin. $\mathbf{b}$ The resultant defect was reconstructed using a Prolene mesh and a latissimus dorsi flap elevated to cover the mesh. c A post-operative clinical photograph. d Clinical photograph 6 months following completion of therapy
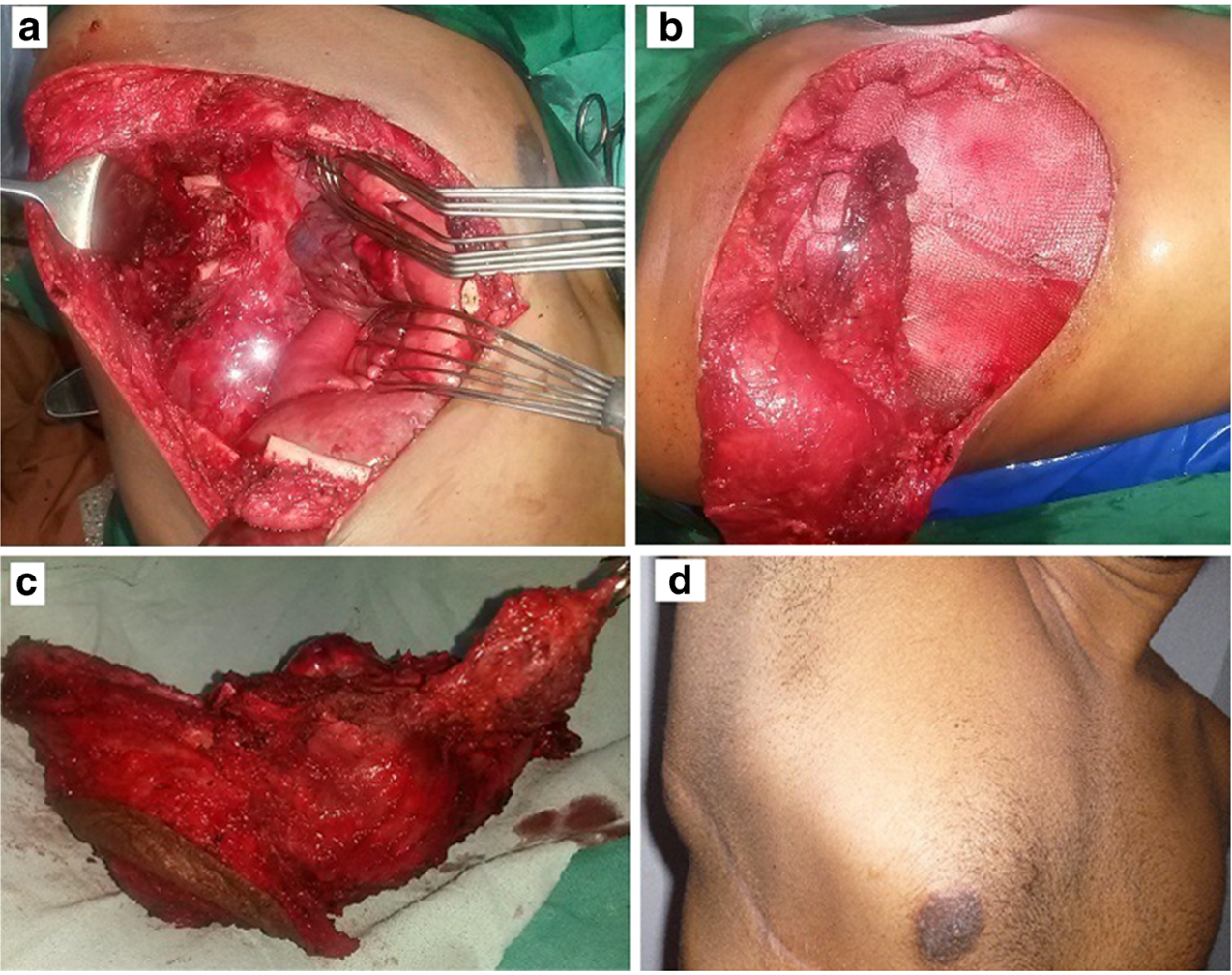

The management strategies for osteosarcomas are multimodal and generally consist of a combination of chemotherapy and surgery. The guidelines for management of a rib primary osteosarcoma is not clear due to the small number of cases studied. Wide en bloc resection aimed at achieving tumour-free margins is necessary in all cases of malignant chest wall tumours including osteosarcomas [5]. Surgery for primary rib osteosarcomas should include resection of the involved ribs with wide margins that may include the adjacent ribs, intercostal muscles and pleura as clinically indicated. The chest wall stability is generally achieved by reconstruction of rib cage with synthetic meshes such as polypropylene or polytetrafluroethylene with or without methyl methacrylate reinforcement. The other options of chest wall reconstruction include the use of titanium meshes, metallic implants and allografts. A full-thickness resection generally precludes a primary closure of the chest wall defect; regional myocutaneous flaps like the latissimus dorsi, rectus abdominis or the pectoralis major have been used to achieve a skin and soft tissue cover.

Multi-agent chemotherapy has been used in many series in an attempt to improve the patients' disease free and overall survival, by reducing the risk of both local and distant relapse. Neoadjuvant chemotherapy is considered as an acceptable modality of treatment in cases wherein the pre-operative diagnosis of osteosarcoma is established [7, 8]. Neoadjuvant chemotherapy was considered in our patient in view of the incomplete resection and the presence of an exudative pleural effusion. Osteosarcomas have traditionally been considered to be relatively radio-resistant tumours. Radiotherapy however has been tried in cases of osteosarcomas of head and neck region and in osteosarcomas involving the axial skeleton especially in cases with positive histological margins or in cases wherein a complete resection is not deemed feasible. The overall prognosis of osteosarcoma in flat bones remains poor despite aggressive surgery and multi-agent chemotherapy; an earlier series had reported a 5 -year survival of only about $27 \%$ $[9,10]$.

In conclusion, this case highlights the fact that osteosarcoma of the rib, although rare, should be considered in the differential diagnosis of primary malignant neoplasms of the rib even in the adult population.

\section{Compliance with Ethical Standards}

Conflict of Interest The authors declare that they have no conflict of interest.

Source of Funding None to declare.

Ethical Issues All procedures performed in this case report were in accordance with the ethical standards of the institutional research committee and with the 1964 Helsinki declaration and its later amendments or comparable ethical standards. 
Informed Consent Appropriate informed consents have been obtained.

\section{References}

1. Mohanty S, Inchara YK, Crasta JA, Ananthamurthy A (2010) An unusual case of primary osteosarcoma of the rib in an adult. Indian $\mathrm{J}$ Med Paediatr Oncol 31:18-20

2. Ikeda H, Takeo M, Kayata H, Mikami R, Nakamoto Y, Yamamoto M (2014) A case of rapidly growing osteosarcoma of the rib. Ann Thorac Cardiovasc Surg 20:521-524

3. Ludhani PM, Anathakrishnan R, Chandrasekar P, Muralidharan S (2013) Unusual case of chondroblastic osteosarcoma of the rib in an adult. Asian Cardiovasc Thorac Ann 22:745-747

4. Chattopadhyay A, Nagendhar Y, Kumar V (2004) Osteosarcoma of the rib. Indian J Pediatr 71:543-544
5. Xie P, Huang $\mathrm{J}$ (2016) Primary osteosarcoma of the rib identified on bone scintigraphy. Clin Nucl Med 41:390

6. Anoop TM, Geetha N, Babanrao SA, Jayasree K (2014) Primary osteosarcoma of rib mimicking lung mass with secondary aneurysmal bone cyst formation. J Thorac Oncol 9:738-739

7. Xu G, Zheng K (2013) Successful management of early recurrence after surgery for primary rib osteosarcoma in an adult. Interact Cardiovasc Thorac Surg 17:431-432

8. Kempf-Bielack B, Bielack SS, Jürgens H, Branscheid D, Berdel WE, Exner GU et al (2005) Osteosarcoma relapse after combined modality therapy: an analysis of unselected patients in the Cooperative Osteosarcoma Study Group (COSS). J Clin Oncol 23:559-568

9. Misiak P, Jabłoński S (2015) High-grade surface osteosarcoma of the rib-a case report. Pol Merkur Lekarski 39:40-42

10. Deitch J, Crawford AH, Choudhury S (2003) Osteogenic sarcoma of the rib: a case presentation and literature review. Spine 28:E74 E77 